\title{
Correlation Between Adiponectin, Tumor Necrosis Factor- $\alpha$, Insulin Resistance and Atherogenic Dyslipidemia in Non Diabetic Central Obese Males
}

\author{
Candra Ninghayu ${ }^{1,2}$, Andi Wijaya ${ }^{1,2}$, and Suryani As'ad ${ }^{3}$ \\ ${ }^{1}$ Prodia Clinical Laboratory \\ ${ }^{2}$ Postgraduate Program in Clinical Biochemistry, Hassanuddin University, Makassar \\ ${ }^{3}$ Faculty of Medicine, Hasanuddin University, Makassar
}

\section{Abstract}

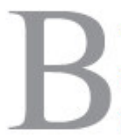

ACKGROUND: Obesity raises the risk for atherosclerotic cardiovascular disease (ASCVD) through many risk factors including atherogenic dyslipidemia. Atherogenic dyslipidemia is characterized by high levels of triglyceride, increased small dense lowdensity lipoprotein particles, and reduced levels of highdensity lipoprotein cholesterol. The exact mechanisms of central obesity and this atherogenic lipoprotein phenotype (ALP) is not clearly understood. Central obesity is characterized by a state of systemic low grade inflammation and insulin resistance. Adipose tissue has recently been shown to secrete a variety of bioactive peptides, called adipocytokines, that can potentially affect glucose and lipid metabolism. The aim of this study was to observe the role of adiponectin, tumor necrosis factor- $\alpha$ (TNF- $\alpha$ ) and insulin resistance in atherogenic dyslipidemia in nondiabetic central obese males.

METHODS: This was a cross-sectional study on 75 non-diabetic central obese male subjects (waist circumferences $>90 \mathrm{~cm}$ ). Adiponectin and TNF- $\alpha$ testing were performed by ELISA; insulin resistance was assessed by the Homeostasis Model Assessment (HOMA) index, triglyceride was assessed by GPO-PAP, HDL cholesterol and small dense LDL were measured by homogenous method. Statistical analysis was done by SPSS for Windows v. 11.5 with a significance level at $\mathrm{p}<0.05$.The Pearson and Spearman's Rho correlation coefficient was used to assess the correlation between various anthropometric and biochemical parameters.

RESULTS: There were 75 patients aged $38.0 \pm 6.3$ years, Adiponectin concentration was $3.55 \pm 1.38 \mu \mathrm{g} / \mathrm{ml}$, HOMA index was $2.28 \pm 1.63$, TNF- $\alpha$ was $12.42 \pm 11.25 \mathrm{pg} / \mathrm{ml}$, triglyceride was $185.17 \pm 109.00$, HDL-cholesterol was $44.15 \pm 9.23 \mathrm{mg} / \mathrm{dL}$, small dense LDL $23.22 \pm 12.26$ $\mathrm{mg} / \mathrm{dL}$. This study revealed that there were correlations between adiponectin and triglyceride $(r=-0.236, p=0.042)$, adiponectin and HDL cholesterol $(r=0.300, p=0.009)$, adiponectin and atherogenic dyslipidemia $(\mathrm{r}=-0.256, \mathrm{p}=$ 0.027 ), whereas there was no correlation between insulin resistance and TNF- $\alpha$ with the atherogenic dyslipidemia.

CONCLUSION: Adiponectin might contribute to atherogenic dyslipidemia in central obese non-diabetic males. Advancing our understanding of the function and measurement of adiponectin serum concentration will be useful in clinical diagnosis of obesity related atherogenic dyslipidemia.

KEYWORDS: Obesity, Waist Circumference, Adiponectin, Insulin Resistance, TNF- $\alpha$, Atherogenic dyslipidemia. 
Introduction

Obesity has reached an epidemic state globally, with more than 1 billion adults overweight-at least 300 million of them obese, and this is a major contributor to the global burden of chronic disease and disability. Often coexisting with under-nutrition in developing countries, obesity is a complex condition with serious social and psychological dimensions affecting virtually all ages and socioeconomic groups (WHO, 2006). A study done by Indonesian Society for the Study of Obesity (ISSO 2004) with more than 6.000 subjects involved, has shown that the prevalence of obesity increased by $9.16 \%$ in man and by $11.02 \%$ in woman. The incidence will increase and can be epidemic 20 years from now if it is not dealt with properly.

Obesity increases the risk of atherosclerotic cardiovascular disease (CVD), i.e. increased triacyl glycerols and small dense low density lipoprotein (sdLDL) particles, and decrease in high density lipoprotein cholesterol (HDL-C). Atherogenic dyslipidemia (atherogenic lipoprotein phenotype), which clinically represented by elevated serum triglyceride levels, increased sdLDL particles, and decreased levels of HDL-C associated with excess adiposity, is highly correlated with reduced insulin sensitivity and is a major feature of the central obesity (1).

Kinetic studies have consistently demonstrated that obesity and insulin resistance are associated with increased production of very low density lipoprotein - triglyceride (VLDL-TG), and in some cases, VLDL apoB. Thus, the mechanisms leading to VLDL overproduction in obesity and insulin resistance are of great interest. Increased VLDL production may be related to increased concentration of free fatty acids in obese patients, which accumulate in the liver and enhance formation of triglyceride-enriched VLDL. Whereas some obese individuals respond to a greater VLDL production by increasing their lypolisis of VLDL triglycerides, other patients develop hypertriglyceridemia. High circulating concentrations of VLDL leads to increased exchange of VLDL triglycerides for cholesterol esters in HDL and LDL by cholesteryl ester transfer protein (CETP). This bidirectional exchange reduces HDL-cholesterol concentration, engenders cholesterol depleted LDL (small dense LDL) and increases the cholesterol content of VLDL. Obese patients also have greater activity of hepatic triglycerides lipase; this mechanism contributes to the decrease of HDL cholesterol concentrations commonly encountered in obese patients $(2,3)$.
The precise mechanism of dyslipoproteinemia, adiposity, and insulin resistance is complex and not yet clearly defined. Adipose tissue has recently been shown to secrete a variety of bioactive peptides, called adipocytokines, that can potentially affect glucose and lipid metabolism. The adipocytokines include adiponectin, leptin, resistin, interleukin-6 (IL-6), and tumor necrosis factor- $\alpha$ (TNF- $\alpha)$. Adiponectin, also known as adipocyte complement-related protein of $30 \mathrm{kDa}$ (ACRP30), adipoQ, and gelatin-binding protein of $28 \mathrm{kDa}$ (GBP28), is a protein present at relatively high concentrations in the circulation. Unlike other adipocytokines, plasma adiponectin concentrations are decreased in obese and insulin-resistant individuals, including those with type 2 diabetes. Experimental and clinical evidence suggests that other adipocytokines may exert their effects on insulin sensitivity by influencing adipocyte expression and secretion of adiponectin . Hypertriglyceridemia, low HDLcholesterol concentrations, and decreased LDL particle size have recently been shown in humans to be correlated with low plasma adiponectin concentrations independent of the amount of intraabdominal fat and degree of insulin resistance. (4).

Adiponectin enhances insulin sensitivity and improves the serum lipid profile through AMPK activation and increased Fatty Acid (FA) oxidation. Conversely, disruption of adiponectin expression causes insulin resistance. TNF- $\alpha$ may therefore induce systemic insulin resistance and dyslipidaemia by suppressing the production of adiponectin; indeed, circulating levels of adiponectin inversely correlate with plasma levels of TNF- $\alpha$.

Familial combined hyperlipidemia $(\mathrm{FCH})$ is the most common genetic hyperlipidemia in humans, affecting $1-3 \%$ of the general population. It is strongly associated with premature cardiovascular disease. From the survivors of premature myocardial infarction, it is known up to $20 \%$ are affected with FCH. Van der Fleuten et al indicate that low adiponectin levels contribute to the atherogenic lipid profile in $\mathrm{FCH}$, independent of insulin resistance and obesity as measured by waist circumference, supports the hypothesis that there is a role of a disturbed adipose tissue metabolism in the pathophysiology of $\mathrm{FCH}$ (6).

Matsubara et al have demonstrated high triglyceride and low HDL cholesterol are associated with low plasma adiponectin concentration in nondiabetic women. Further studies must now be carried out to determine whether it is partly responsible for the atherogenic risk (7).

The aim of this study was to observe the role of adiponectin, tumor necrosis factor- $\alpha$ (TNF- $\alpha$ ) and insulin resistance in the atherogenic dyslipidemia. 


\section{Methods}

This study was a cross-sectional design involving 75 nondiabetic central obese male subjects, as defined by waist circumference $>90 \mathrm{~cm}$ (according to the International Diabetes Federation Criteria/IDF criteria) and fasting plasma glucose $<126 \mathrm{mg} / \mathrm{dL}$ (according to Perkumpulan Endokrinologi Indonesia/PERKENI criteria). Subjects with hypertension (blood pressure $>140 / 85 \mathrm{mmHg}$ ), liver failure, renal failure, and who had consumed anti-inflammatory drug in the last 3 months or had an acute inflammation were excluded from the study. Anthropometric measurements (height, weight, BMI, waist circumference, and blood pressure) and routine biochemical variables (fasting glucose, fasting insulin, triglycerides, HDL-cholesterol, creatinine, total bilirubin, aspartate aminotransferase and alanine aminotransferase) were measured on all subjects. Insulin resistance was estimated with the homeostasis model assessment index (HOMA-IR), calculated as fasting glucose (in $\mathrm{mmol} / \mathrm{L}$ ) times fasting insulin (in $\mathrm{mIU} / \mathrm{L}$ ) divided by 22.5 .

Adiponectin was assayed by sandwich enzyme-linked immunosorbent assay (ELISA) (Sekisui Medical Co Ltd, Japan). hs-TNF- $\alpha$ was measured by sandwich enzyme immunoassay technique from R\&D Systems Inc, USA. sdLDL was measured by homogenous method from Denka Seiken, Tokyo, Japan.

All statistical calculations were done with SPSS 11.5 statistical software package. Univariate analysis was carried out to calculate mean, maximum and minimum value and $\mathrm{SD}$. We used non parametric analysis of Kolmogorof - Smirnov to assess whether each variable was normally distributed or not. The association between various measurements were analyzed with Pearson correlation when the data were normally distributed and with Spearman's rho correlation when the data were not normally distributed. We considered $\mathrm{p}<0,05$ as significant correlation.

\section{Results}

The characteristics of the subject, measurements, height, weight, BMI, waist circumference, and blood pressure, fasting glucose, fasting insulin, triglycerides, HDL-cholesterol, creatinine, bilirubin, aspartate aminotransferase (AST) and alanine aminotransferase (ALT) and measurement of adiponectin, hs TNF- $\alpha$, sd LDL are shown in Table 1.

Table 1. Baseline Characteristics of the Study Subjects

\begin{tabular}{lcc}
\hline PARAMETERS & Mean & SD \\
$\mathbf{N}=75$ & & \\
\hline Age $($ years $)$ & 38.00 & 6.30 \\
Waist circumference $(\mathrm{cm})$ & 98.42 & 8.04 \\
Height $(\mathrm{cm})$ & 167 & 0.07 \\
Weight $(\mathrm{kg})$ & 82.03 & 15.94 \\
Body Mass Index $(\mathrm{kg} / \mathrm{m} 2)$ & 29.33 & 5.12 \\
Systolic Blood Pressure $(\mathrm{mmHg})$ & 115.40 & 7.79 \\
Diastolic Blood Pressure $(\mathrm{mmHg})$ & 77.00 & 6.04 \\
AST $(\mathrm{U} / \mathrm{L})$ & 26.88 & 6.51 \\
ALT $(\mathrm{U} / \mathrm{L})$ & 38.16 & 13.60 \\
Total bilirubin $(\mathrm{mg} / \mathrm{dl})$ & 0.76 & 0.24 \\
Creatinine $(\mathrm{mg} / \mathrm{dl})$ & 0.94 & 0.16 \\
Fasting Blood Sugar $(\mathrm{mg} / \mathrm{dL})$ & 86.00 & 9.53 \\
Insulin $(\mu \mathrm{lU} / \mathrm{mL})$ & 10.37 & 6.12 \\
HOMA-IR & 2.28 & 1.63 \\
Adiponectin $(\mu \mathrm{gg} / \mathrm{mL})$ & 3.55 & 1.38 \\
TNF- $\alpha(\mathrm{pg} / \mathrm{mL})$ & 12.42 & 11.26 \\
Triglyceride $(\mathrm{mg} / \mathrm{dL})$ & 185.17 & 109.00 \\
HDL-Cholesterol $(\mathrm{mg} / \mathrm{dL})$ & 44.15 & 9.23 \\
sdLDL $(\mathrm{mg} / \mathrm{dL})$ & 23.22 & 12.26 \\
& &
\end{tabular}


Data grouping of low concentration and high concentration of adiponectin was based on $2.54 \mu \mathrm{g} / \mathrm{mL}$ and HOMA IR grouping was based on $4(9)$. TNF- $\alpha$ data grouping was based on median: lower concentration group and higher concentration. In this study, there was correlation between Adiponectin with triglycerides $(r=-0.236, \mathrm{p}=0.042$ ) and HDL-cholesterol ( $\mathrm{r}=0,300, \mathrm{p}=0.009)$, whereas there was no correlation between adiponectin and small dense
LDL. No significant correlation was observed between HOMA-IR and TNF- $\alpha$ and triglycerides, HDL-cholesterol and small dense LDL. This study also showed that there was no correlation between HOMA-IR and TNF- $\alpha$ with atherogenic dyslipidemia.

The results in Fig. 1 shows that there were weak but a significant negative correlation between adiponectin with atherogenic dyslipidemia $(\mathrm{r}=-0,256, \mathrm{p}=0.027)$.

\section{Table 2. Correlation among Variables}

\begin{tabular}{|c|c|c|c|c|c|c|}
\hline \multirow[t]{2}{*}{ Variable } & \multicolumn{2}{|c|}{ Triglyceride } & \multicolumn{2}{|c|}{ HDL-Cholesterol } & \multicolumn{2}{|c|}{ sdLDL } \\
\hline & $\mathbf{r}$ & $p$ & $r$ & $\mathbf{p}$ & $r$ & $\mathrm{p}$ \\
\hline Adiponectin & -0.236 & $0.042^{\star}$ & 0.300 & $0.009^{\star \star}$ & -0.167 & 0.152 \\
\hline HOMA-IR & 0.197 & 0.092 & -0.038 & 0.746 & 0.069 & 0.557 \\
\hline TNF- $a$ & -0.150 & 0.243 & 0.177 & 0.170 & -0.296 & 0.020 \\
\hline
\end{tabular}

${ }^{*} \mathrm{p}<0.05,{ }^{* \star} \mathrm{p}<0.01$

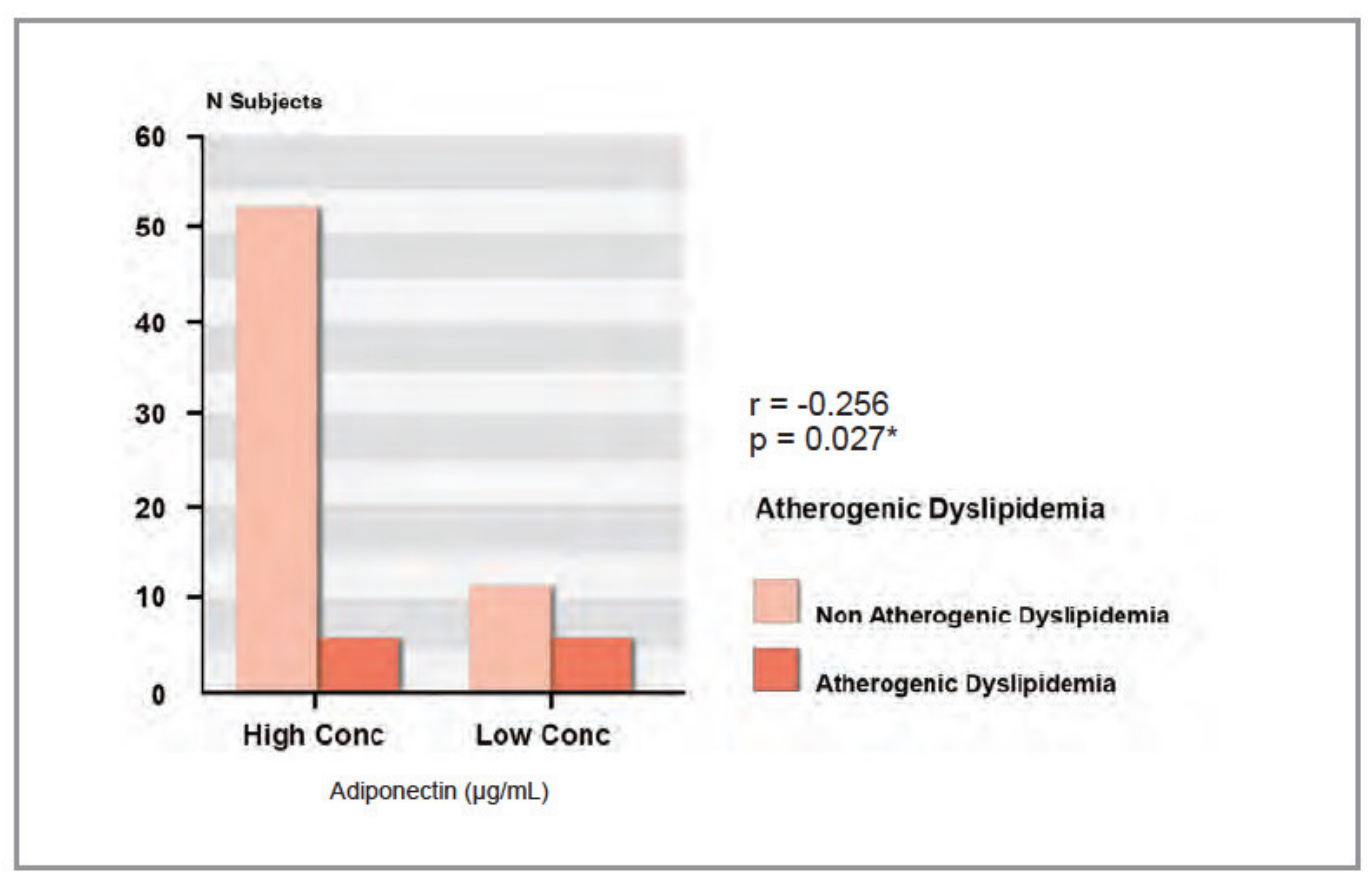

Fig 1. Correlation Between Adiponectin and Atherogenic Dyslipidemia. 


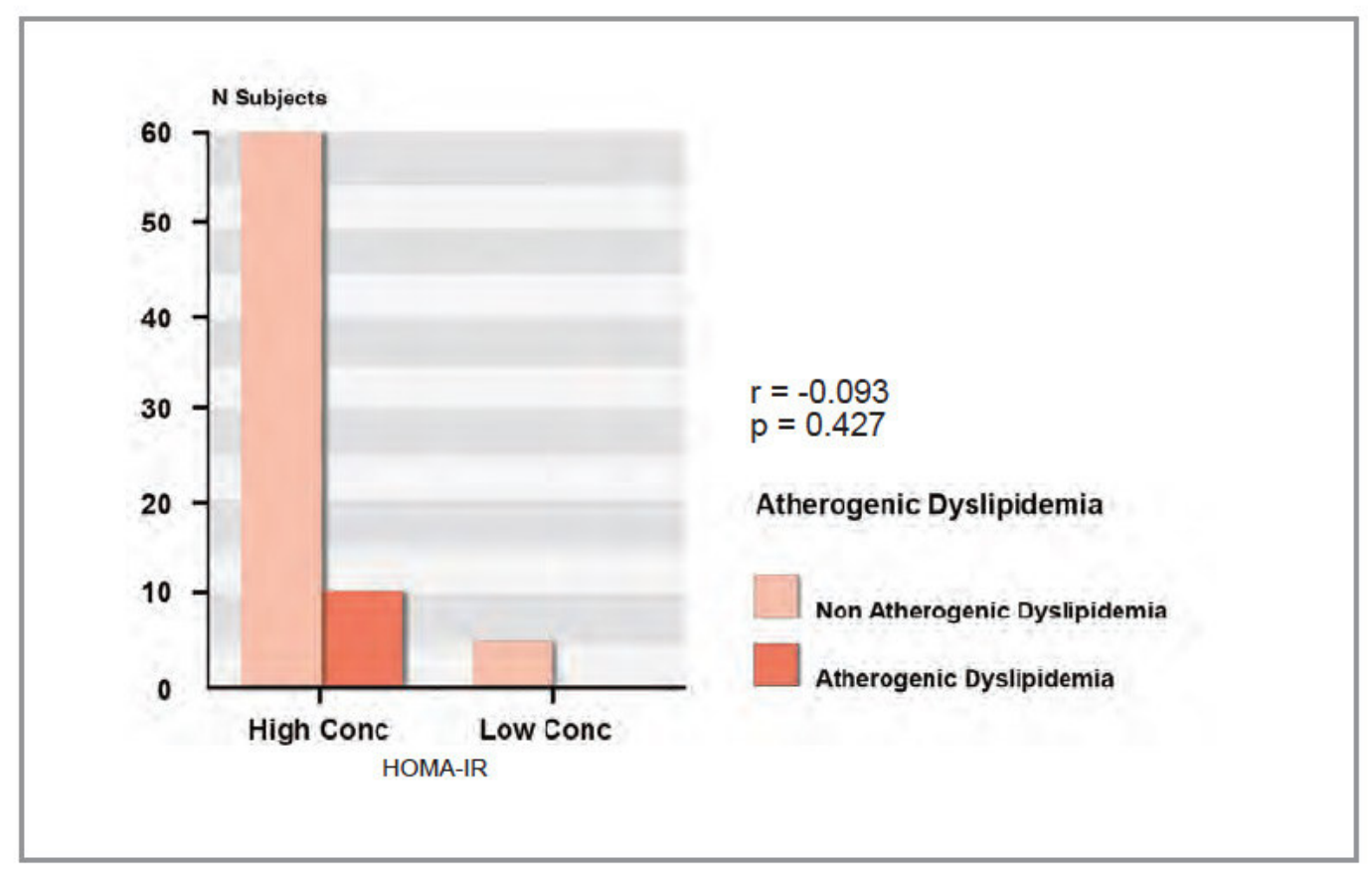

Fig 2. Correlation Between HOMA-IR and Atherogenic Dyslipidemia.

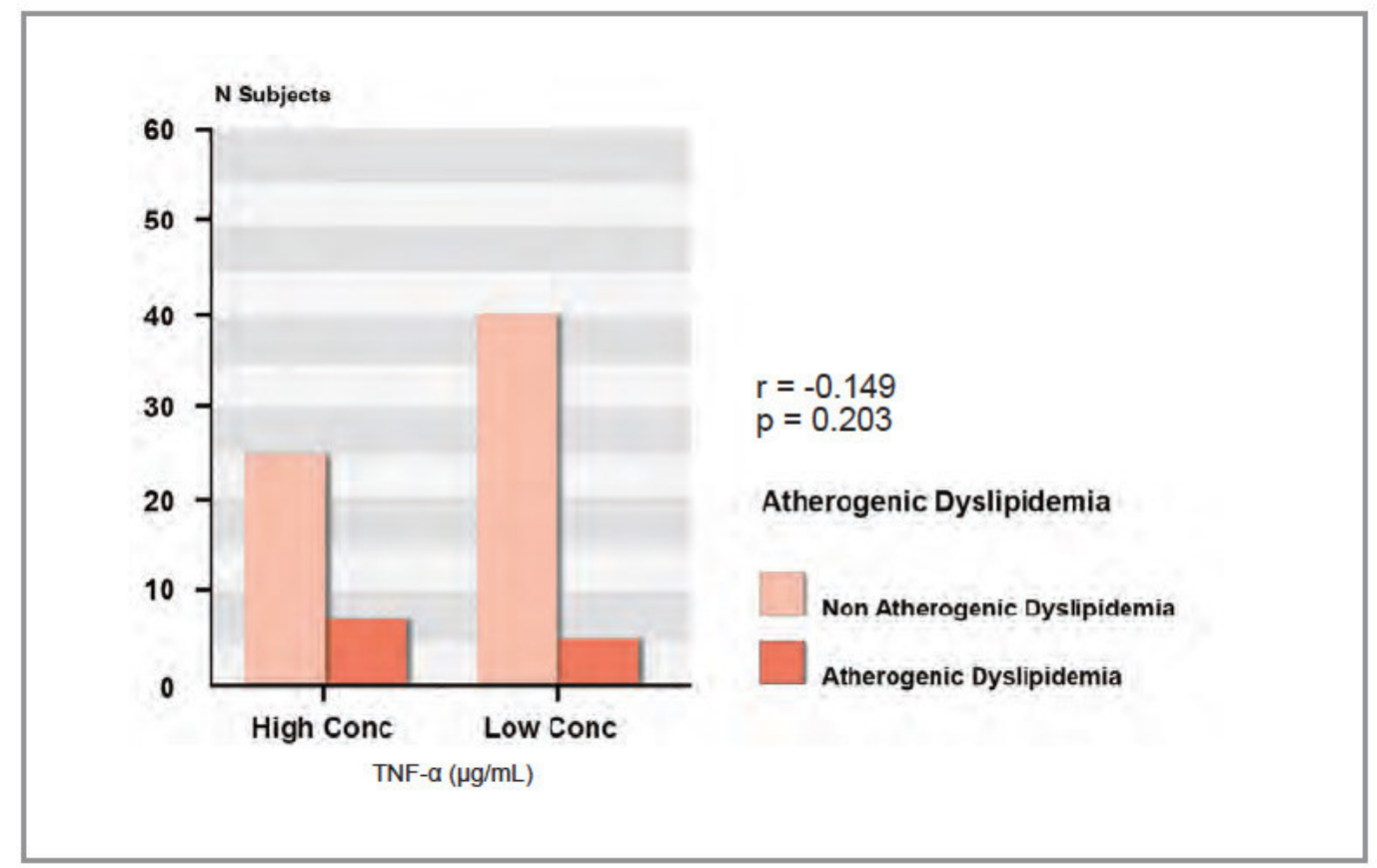

Fig 3. Correlation Between TNF- $\alpha$ and Atherogenic Dyslipidemia. 


\section{Discussion}

The present study demonstrated that in 75 male subjects with central obesity, we found 17 (22.67\%) subjects with hypoadiponectinemia. Low adiponectin concentration showed a significant negative correlation with atherogenic dyslipidemia. Furthermore, association between adiponectin and atherogenic dyslipidemia was independent of insulin resistance and TNF- $\alpha$; adiponectin had a negative correlation with triglycerides and a positive correlation with HDL cholesterol levels. Dyslipidemia in obesity is fundamentally related to expansion in the plasma pool of triglyceride-rich lipoprotein (TRL). The effect of adiponectin on TRL metabolism may principally involve intrinsic changes in skeletal muscle lipid metabolism and effects on lipoprotein lipase activity in both skeletal muscle and adipocytes. Adiponectin may decrease accumulation of triglycerides in skeletal muscle by enhancing fatty acid oxidation through activation of acetylCoA oxidase, carnitine palmitoyltransferase-1, and AMP kinase. Adiponectin may also stimulate both lipoprotein lipase (LPL), the lipolytic enzyme that catabolizes VLDL, and apoC-III by increasing the expression of peroxisome proliferator-activated receptor- $\alpha$ in the liver and adipocytes. Von Eynatten $\mathrm{M}$ et al have found that the relationship between adiponectin and postheparin LPL activity is independent of both adiponectin effects on whole-body insulin sensitivity and systemic inflammation. Adiponectin's anti-inflammatory properties may directly stimulate the expression of $\operatorname{LPL}(9,10)$.

The relationship between adiponectin and plasma lipoprotein could be mediated by the effects of adiponectin on hepatic lipase activity, which increases in central obesity and insulin resistance states. While hepatic lipase does not seem to be acutely regulated by insulin, but it could be increased in reduced adiponectin concentrations (11).

There was no significant correlation found between HOMA-IR and atherogenic dyslipidemia . Our data also showed that there was no significant correlation between TNF- $\alpha$ and atherogenic dyslipidemia.

According to Sethi JK and Vidal-Puig A.J. proinflammatory cytokines play an important role in both immunity and energy metabolism. In obesity, the inappropriate but chronic increase in proinflammatory cytokine production in adipose tissue can have a profound impact on the whole body energy balance. The negative action of the prototypical cytokine TNF- $\alpha$ on insulin sensitivity has been well documented. However, TNF- $\alpha$ also inhibits adipocyte differentiation that promotes lipid mobilization in mature adipocytes and impairs the function of brown adipose tissues. Many of these actions are mediated by the same TNF receptor 1 , suggesting that some signaling specificity exists. Together, these actions effectively limit adipose tissue expandability and decrease its lipid storage capacity. In this way, local TNF action in adipose tissue can significantly contribute to obesityassociated hyperlipidemia and lipotoxicity in other organs such as muscles, liver, and b-cells (12). Most subjects of this study were bellow 40 years of age, thus we assume that the inflammation and insulin resistance were of the early stage, therefore their effects had not yet been obvious.

\section{Conclusions}

In conclusion, adiponectin might contribute to atherogenic dyslipidemia in central obese non-diabetic males. Advancing our understanding on the function and measurements of adiponectin serum concentration will be useful for the clinical diagnosis of obesity-related atherogenic dyslipidemia.

\section{Acknowledgements:}

We are thankful for the support and funding given by Prodia Research Foundation.

\section{References:}

1. Krauss R.M., Blanche P. J., Rawlings R. S., Fernstrom H. S, and Williams P. T. Separate Effects of Reduced Carbohydrate Intake and Weight Loss on Atherogenic Dyslipidemia. J Clin Nutr 2006; 83 : 1025-31.

2. Bamba V. and Rader D. J. Obesity and Atherogenic Dyslipidemia. Gastroenterology 2007; 132 : 2181-2190.

3. Grundy S. M., Abate N. Obesity. In: Secondary Heart Disease:Systemic Disease and The Heart: 2nd ed; 2002. p. $1463-68$

4. Chan D. C., Watts G. F., Ng T. W.K., et al. Adiponectin and other Adipocytokines as Predictors of Markers of Triglyceride-Rich Lipoprotein Metabolism. Clin Chem 2005; 51: 578-585

5. Cawthorn W.P., Sethi J.K., TNF- $\alpha$ and Adipocyte Biology. Febs Lett 2008, 582 :117-131

6. Van der Vleuten G. M., van Tits L. J. H, den Heijer M., Lemmers H., Stalenhoef A. F. H., and de Graaf J. Decreased adiponectin levels in familial combined hyperlipidemia patients contribute to the atherogenic lipid profile. J. Lipid Res 2005; 46 : 2398-2404.

7. Matsubara M., Maruoka S., and Katayose S. Decreased Plasma Adiponectin Concentrations in Women with Dyslipidemia. J Clin Endocrinol Metab 2002; 87 : 27642769 
8. Ebinuma H., Miyazaki O., Yago $H_{\text {., }}$ Hara K., Yamauchi T., Kadowaki T., A Novel ELISA system for Selective Measurement of human Adiponectin Multimers by Using Proteases.Clin Chem Acta 2006; 20 : 1-7

9. Chan D. C. Barrett P. H. R, Ooi E. M. M, Ji J., Chan D. T., and Watts G. F. Very Low Density Lipoprotein Metabolism and Plasma Adiponectin as Predictors of High-Density Lipoprotein Apolipoprotein A-I Kinetics in Obese and Nonobese Men, J Clin Endocrinol Metab 2009; 94 : 989997

10. Von Eynatten M, Hamann A., Twardella D., Nawroth PP. Brenner H., and Rothenbacher D. Relationship of
Adiponectin with Markers of Systemic Inflammation, Atherogenic Dyslipidemia, and Heart Failure in Patients with Coronary Heart Disease, Clin Chem 2006; 52 : 853 859

11. Cnop M., Havel P.J., Utzschneider K. M., Carr D. B.,et al. Relationship of Adiponectin to Body Fat Distribution, Insulin Sensitivity and Plasma Lipoproteins: Evidence for Independent Roles of Age and Sex, Diabetologia 2003; 46 : $459-469$

12. Sethi J. K., Puig A. J. V. Adipose Tissue Function and Plasticity Orchestrate Nutritional Adaptation. J Lipid Res 2007; 48 : $1253-1262$ 